\title{
A MULTILAYER APPROACH TO FABRICATE BIOACTIVE GLASS COATINGS ON TI ALLOYS
}

\author{
J.M. GOMEZ-VEGA*, E. SAIZ*, A.P. TOMSIA*, G.W. MARSHALL**, S.J. MARSHALL** \\ *Materials Science Division. Lawrence Berkeley National Laboratory. Berkeley CA 94720. \\ USA \\ **Department of Restorative Dentistry. University of California. San Francisco CA 94143. USA
}

\section{ABSTRACT}

Glasses in the system Si-Ca-Na-Mg-P-K-O with thermal expansions coefficients close to that of Ti6Al4V were used to coat the titanium alloy by a simple enameling technique. Firings were done in air at temperatures between 800 and $840^{\circ} \mathrm{C}$ and times up to 1 minute. Graded compositions were obtained by firing multilayered glass coatings. Hydroxyapatite (HA) particles were mixed with the glass powder and the mixture was placed on the outer surface of the coatings to render them more bioactive. Coatings with excellent adhesion to the substrate and able to form apatite when immersed in a simulated body fluid (SBF) can be fabricated by this methodology.

\section{INTRODUCTION}

Titanium-based alloys, especially Ti6Al4V, are widely used for the fabrication of implants which undergo high stress, such as hip implants. ${ }^{1}$ Nevertheless, the adherence of these implants to the bone is very low, so the utilization of coatings has been investigated as a way to improve their adhesion. ${ }^{2}$ The most studied approach has been the application of hydroxyapatite (HA) coatings by plasma spray and ion sputtering. ${ }^{3,4}$ However, both techniques have failed to produce reliable and long lasting coatings due to weak metal/HA interfaces. Moreover, the high temperature utilized in the plasma spray technique, which gives rise to partial decomposition of $\mathrm{HA}$, and the excessive compactness of the coatings manufactured by ion sputtering, which hampers the osseointegration, are additional problems associated to these techniques. ${ }^{5,6}$

In a previous work, glasses in the system Si-Ca-Na-Mg-P-K-O were successfully used to coat Ti6Al4V by a conventional enameling technique. ${ }^{7,8}$ Some of these glasses, as well as the coatings fabricated with them, were able to form apatite in vitro. ${ }^{9}$ This work explores a multilayer approach to fabricate graded coatings in order to improve their bioactivity, at the same time that good adhesion to the substrate is maintained.

\section{EXPERIMENTAL}

The composition of the glasses used in this research was tailored to obtain thermal expansion coefficients close to that of Ti6Al4V $\left(9.6 \cdot 10^{-6}{ }^{\circ} \mathrm{C}^{-1}\right)$, as well as adequate softening points for enameling below the $\alpha \rightarrow \beta$ transformation of $\mathrm{Ti}$ in the alloy $\left(\sim 980^{\circ} \mathrm{C}\right)$. The glasses were prepared by mixing and firing the required reagents using a standard procedure. ${ }^{7}$ The glasses and HA (99\% purity) were ground to a particle size $<20 \mu \mathrm{m}$ in an agate mortar. The composition and main thermal properties of the glasses used in this work are listed in Table I. Ti6A14V (99\% purity) plates $(15 \times 10 \times 1 \mathrm{~mm})$ were ground with silicon carbide paper ( 800 grit) and cleaned with acetone and ethanol previously to their utilization. To manufacture the coatings, a glass suspension in ethanol was poured onto the plates placed in a beaker, and it was allowed to deposit for around 3 hours. A different suspension was gently poured over the 
remaining clear liquid to deposit a second layer. This operation was repeated as many times as the desired number of layers. Afterwards, the remaining liquid was evaporated at $75^{\circ} \mathrm{C}$ overnight. The green-coatings prepared as indicated were fired in air in a Unitek dental furnace according to the optimum conditions for the glass in contact with the metal (furnace preheating to $600^{\circ} \mathrm{C}$, temperature ramp $40^{\circ} \mathrm{C} / \mathrm{min}$ in low vacuum, final temperature $800-840^{\circ} \mathrm{C}$, dwell time $30 \mathrm{~s}$, and quenching). ${ }^{10}$

Table I Composition and main properties of the synthesized glasses

\begin{tabular}{|c|c|c|c|c|c|c|c|c|}
\hline & \multicolumn{6}{|c|}{ Composition (wt $\%$ ) } & \multirow{2}{*}{$\begin{array}{c}\alpha \\
\left(10^{-6}{ }^{\circ} \mathrm{C}^{-1}\right) \\
\end{array}$} & \multirow{2}{*}{$\begin{array}{c}\text { Ts } \\
\left({ }^{\circ} \mathrm{C}\right) \\
\end{array}$} \\
\hline & $\mathrm{SiO}_{2}$ & $\mathrm{Na}_{2} \mathrm{O}$ & $\mathrm{CaO}$ & MgO & $\mathbf{P}_{2} \mathbf{O}_{5}$ & $\mathbf{K}_{2} \mathbf{O}$ & & \\
\hline 6P50 & 49.8 & 15.5 & 15.6 & 8.9 & 6.0 & 4.2 & 12.2 & 560 \\
\hline 6P53 & 52.6 & 10.4 & 18.0 & 10.2 & 6.0 & 2.8 & 11.5 & 608 \\
\hline $6 P 55$ & 54.5 & 12.0 & 15.0 & 8.5 & 6.0 & 4.0 & 11.0 & 602 \\
\hline 6P57 & 56.5 & 11.0 & 15.0 & 8.5 & 6.0 & 3.0 & 10.8 & 609 \\
\hline 6P61 & 61.1 & 10.3 & 12.6 & 7.2 & 6.0 & 2.8 & 10.2 & 624 \\
\hline 6 Р68 & 67.7 & 8.3 & 10.1 & 5.7 & 6.0 & 2.2 & 8.8 & 644 \\
\hline
\end{tabular}

$\alpha=$ Coefficient of thermal expansion (measured between 200 and $400^{\circ} \mathrm{C}$ )

Ts $=$ Softening point

The coating surfaces, as well as polished cross sections, were examined by optical and scanning electron microscopy with associated wavelength dispersive analysis (SEM-WDX). The strength of the glass/metal interfaces was qualitatively evaluated by Vickers indentations on the coating cross sections with $0.2 \mathrm{Kg}$ load in air. The in vitro behavior of the coatings was tested by immersion in $\mathrm{SBF}^{11}$ at $37^{\circ} \mathrm{C}$ for times up to 30 days.

\section{RESULTS}

In a previous work, it was shown that it is possible to coat Ti6Al4V with glasses 6P57, 6P61 and 6P68 by an enameling technique, whereas coatings made with glasses whose silica content is $\leq 55 \mathrm{wt} \%$ always crack due to an excessive difference in the thermal expansion coefficients with the alloy. ${ }^{10}$ The Vickers indentation at the glass/metal interface on the cross sections of 6P68, 6P61 and 6P57 coatings produced cracks which tend to be driven through the glass and never through the interface (Figure 1). However, the capacity of these glasses to precipitate HA when tested in SBF is very limited due to their high silica content, and HA only starts to nucleate on the 6P57 coating after soaking in SBF for 30 days. ${ }^{9}$ The purpose of this work is to use a multilayer approach to fabricate graded coatings with low silica content (higher bioactivity) on their outer surface, maintaining integrity and good adhesion to the metal. The multilayer approach has been also used to add a glass-HA mixture as the outer layer. The coatings fabricated in this work and their results are listed in Table II. 


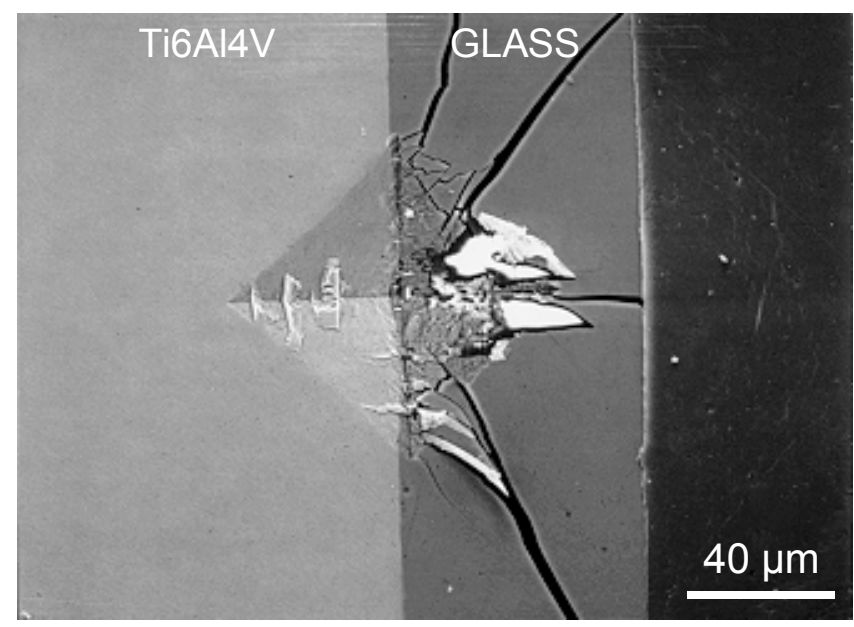

Figure 1 Vickers indentation at the glass 6P57/alloy interface $\left(800^{\circ} \mathrm{C} / 30 \mathrm{~s}\right.$, air $)$ using $0.2 \mathrm{~kg}$ load in ambient air.

Table II Multilayered glass coatings results

\begin{tabular}{lccc}
\hline Glass layers & Thickness $(\mu \mathrm{m})$ & Firing T $\left({ }^{\circ} \mathrm{C}\right)$ & Coating results \\
\hline 6P68/6P55 & $30 / 20$ & 840 & Cracked \\
6P61/6P55 & $30 / 20$ & 820 & Good \\
6P57/6P53 & $30 / 20$ & 800 & Good \\
6P57/6P50 & $30 / 20$ & 800 & Cracked \\
6P57/6P53/6P50 & $30 / 10 / 10$ & 800 & Cracked \\
6P57/(6P57+HA) & $30 / 20 *$ & 800 & Good \\
\hline
\end{tabular}

$* \mathrm{HA}: 6 \mathrm{P} 57=50 \mathrm{wt} \%$

It was not possible to apply $50 \mu \mathrm{m}$ coatings of glasses 6P55 or 6P53 directly on the metal; however, it was possible to prepare coatings containing these glasses when an intermediate glass layer was placed between them and the substrate. Thus, bilayered coatings 6P61/6P55 and 6P57/6P53 could be fabricated. The WDX line profile for Si along 6P61/6P55 coating shows the transition in the silica content between both glass layers (Figure 2). Multilayered coatings with an external layer of glass 6P50 always cracked during cooling. Figure 3 shows a detail of the 6P57/6P50 surface where it can be seen how cracks originated at defects.

A different approach was to deposit a glass-HA mixture as a layer on top of a glass that gives good adhesion to the metal. The cross section of $6 \mathrm{P} 57 /(6 \mathrm{P} 57+\mathrm{HA})$ coating is shown in Figure 4. This coating exhibits a strong glass/metal interface and contains HA particles embedded in the outer layer. Moreover, no reaction product between glass and HA was detected.

When tested in vitro, all the multilayered coatings formed an apatite on the surface after 30 days in SBF. The coating containing embedded HA particles was completely covered by precipitated apatite (Figure 5). 


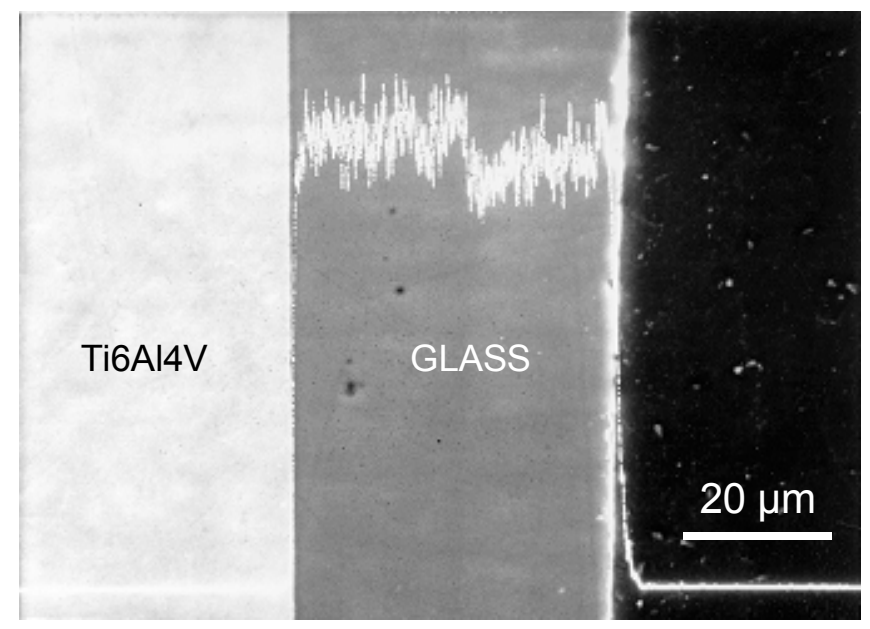

Figure 2 Silicon line analysis (calibrated WDX) along the cross sections of 6P61/6P55 coating.

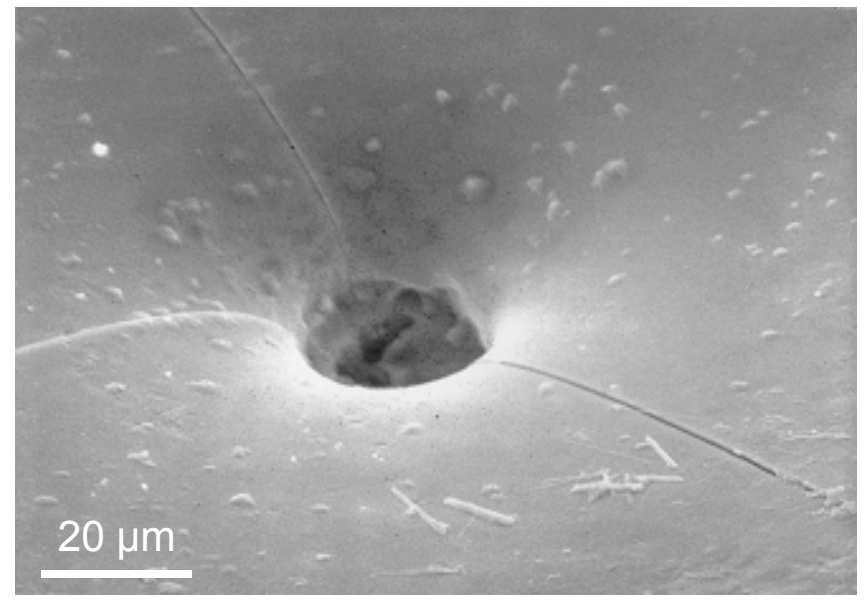

Figure 3 6P57/6P50 coating surface where it can be observed that cracks initiated at defects.

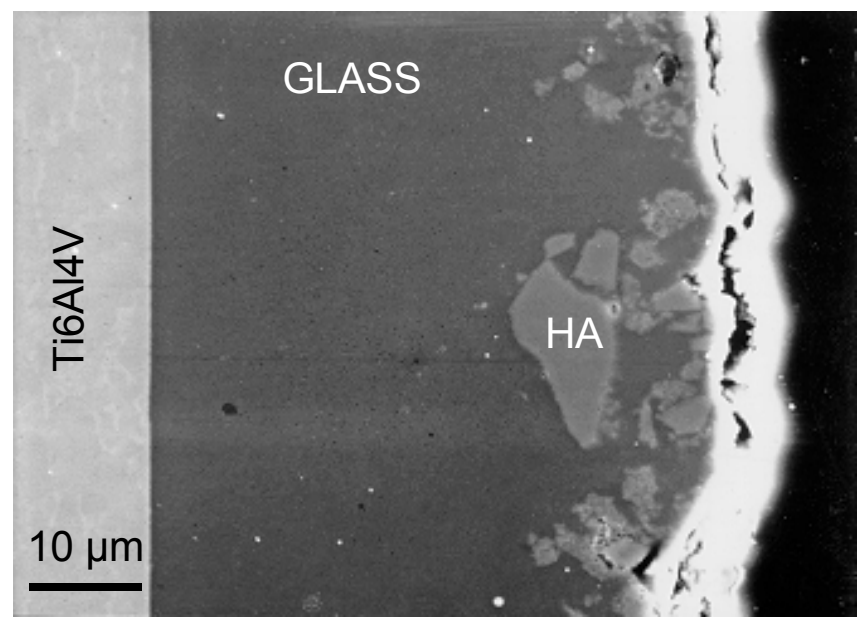

Figure 4 Cross-section of coating 6P57/(6P57+HA). 


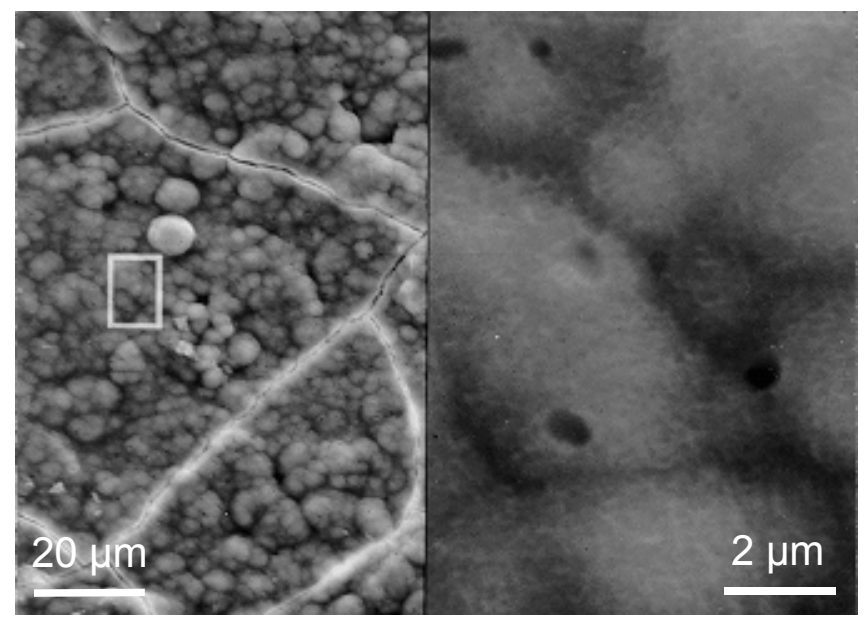

Figure 5 Crystallized hydroxyapatite formed on the surface of the coating 6P57/(6P57+HA) after immersion in SBF for 30 days.

The source of stress in the coatings is the difference in thermal expansion coefficients between the glasses and the metallic substrate. Due to the small thickness of the coating compared to the substrate (50:1000), only a moderate reduction of thermal stress can be expected on the external layer using a multilayered coating, compared to when it is applied directly on the metal. This is the reason why coatings with glass 6P50 were always cracked. Nevertheless, reductions in the edge stresses and some glass interdiffusion are important benefits associated with a multilayer approach. Thus, the silica content on the external layer of the coatings could be reduced with respect to the values attainable in single layer coatings ( 57 to $53 \mathrm{wt} \%$ ). Another advantage of the multilayer approach is the possibility to design coatings with an internal insoluble glass layer that can protect the metallic implant from corrosion, while maintaining a bioactive surface.

When using glass-HA mixtures, two kind of stresses coming from differences in thermal expansion coefficients must be considered (i) between glass and HA (reported $\alpha_{\mathrm{HA}}$ ranges between $11.6-14.0 \cdot 10^{-6}{ }^{\circ} \mathrm{C}^{-1}$ ) and (ii) between the glass-HA composite and the metallic substrate. Using the Turner model (with similar Poisson's ratio for HA and glass), ${ }^{12}$ the overall thermal expansion of the composite (6P57-HA $50 \mathrm{wt} \%$ ) will range between $\alpha=11.2-12.2 \cdot 10^{-6}{ }^{\circ} \mathrm{C}^{-1}$ $\left(\mathrm{d}_{6 \mathrm{P} 57}=2.60 \mathrm{~g} / \mathrm{cm}^{3}, \mathrm{~d}_{\mathrm{HA}}=3.15 \mathrm{~g} / \mathrm{cm}^{3}\right)$. No cracks could be detected either at the glass $/ \mathrm{HA}$ or glass/metal interfaces, so the coating can endure the tension created by the HA particles embedded in the outer surface.

The HA particles enhanced the capacity of the coatings to precipitate apatite when immersed in SBF. It has been also observed that glass coatings with attached HA particles got noticeably richer in $\mathrm{Ca}$ and $\mathrm{P}$ during in vitro tests, which supported the precipitation of apatite on most of its surface. ${ }^{11}$

\section{CONCLUSIONS}

Ti6Al4V can be coated by an enameling technique with a family of silicate based glasses with tailored composition to match the thermal expansion coefficient of the metal. A multilayer glass coating is a successful approach to coatings with excellent adhesion to the substrate and bioactive surface. Coatings with a silica content as low as $53 \mathrm{wt} \%$ on the surface can be fabricated by a double glass layer 6P57/6P53. Coatings with HA particles embedded on the 
outer layer also can be fabricated by this methodology. Apatite precipitated profusely on these coatings after soaking in SBF for periods of 30 days.

Glass-HA and silica functionally graded coatings are accessible by a multilayer technique. This methodology is a promising way to more reliable and long-lasting metallic implants.

\section{ACKNOWLEDGEMENTS}

This work was supported by the NIH/NIDR grant 1R01DE11289. Jose M. Gomez-Vega wishes to thank FICYT for the postdoctoral fellowship given to him in the II Plan Regional de Investigación del Principado de Asturias (Spain).

\section{REFERENCES}

1. L.L. Hench, J. Am. Ceram. Soc., 74 (7), 1487-1510 (1991).

2. T. Kitsugi, T. Nakamura, M. Oka, Y. Senaha, T. Goto and T. Shibuya, J. Biomed. Mater. Res., 30 (2), 261-269 (1996).

3. W. Suchanek and M. Yoshimura, J. Mat. Res, 13 (1), 94-117 (1998).

4. W.R. Lacefield, in An Introduction to Bioceramics, edited by L.L. Hench and J. Wilson (World Scientific, Singapore, 1993) pp. 223-238.

5. S.W. Ha, R. Reber, K.L. Ecjert, M. Petitmermet, J. Mayer, E. Wintermanterl, Ch. Baerlacher and H. Gruner, J. Am. Ceram. Soc., 81 (1), 81-88 (1998).

6. K.A. Gross, V. Gross and C.C. Berdin, J. Am. Ceram. Soc., 81 (1), 106-112 (1998).

7. A. Pazo, E. Saiz and A.P. Tomsia, Acta Mater., 46 (7), 2551-2558 (1998).

8. A. Pazo, E. Saiz and A.P. Tomsia, Ceramic Microstructures, edited by A.P. Tomsia and A.M. Glaeser (Plenum Press, New York, 1998) pp. 543-550.

9. E. Saiz, L. Szebeszczyk, J.M. Gomez-Vega and A.P. Tomsia, J. Biomed. Mater. Res. (submitted).

10. J.M. Gomez-Vega, E. Saiz and A.P. Tomsia, J. Biomed. Mater. Res. (submitted).

11. J.M. Gomez-Vega, E. Saiz and A.P. Tomsia, Biomaterials (submitted).

12. W.D. Kingery, H.K. Bowen, D.R. Uhlman, Introduction to Ceramics, 2nd ed. (John Wiley \& Sons, New York, 1976), p. 604. 